Revue Sciences/Lettres

Sciences / Lettres

1 | 2013

Transferts culturels

\title{
Comment les sciences durent
}

\section{Éric Brian}

\section{(2) OpenEdition}

\section{Journals}

Édition électronique

URL : http://journals.openedition.org/rsl/347

DOI : $10.4000 /$ rsl.347

ISSN : 2271-6246

\section{Éditeur}

Éditions Rue d'Ulm

\section{Référence électronique}

Éric Brian, «Comment les sciences durent », Revue Sciences/Lettres [En ligne], 1 | 2013, mis en ligne le 01 juin 2012, consulté le 01 mai 2019. URL : http://journals.openedition.org/rsl/347 ; DOI : 10.4000/ rsl.347

Ce document a été généré automatiquement le 1 mai 2019.

(C) Revue Sciences/Lettres 


\title{
Comment les sciences durent
}

\author{
Éric Brian
}

1 A-t-on jamais disposé jusqu'à ce jour d'autant de vestiges des activités scientifiques antérieures, d'autant d'instruments qui les conservent et les combinent plus ou moins arbitrairement, d'autant de ressources propres à mettre à l'épreuve et à renouveler l'exercice de la science ? Ce mixte de profusion matérielle et de potentialité technique à peine concevable - porté bien sûr par un essor des technologies de l'information qui n'est pas propre aux sciences elles-mêmes - arrive au moment même où les milieux scientifiques connaissent une cassure démographique rare. La génération entrée dans la carrière au temps de l'expansion de l'Université et des établissements de recherche se retire massivement. De surcroît, cette conjoncture déjà exceptionnelle coïncide avec des bouleversements fondamentaux des modes de financement et des cadres administratifs quotidiens, qui répondent, dit-on, à des exigences de révision des formes du soutien public aux sciences.

Or, pour le dire à la manière d'un sociologue :

Chaque science a, pour ainsi dire, une âme qui vit dans la conscience des savants. Une partie seulement de cette âme prend un corps et des formes sensibles. Les formules qui l'expriment, étant générales, sont aisément transmissibles. Mais il n'en est pas de même dans cette autre partie de la science qu'aucun symbole ne traduit au-dehors. Ici, tout est personnel et doit être acquis par une expérience personnelle. Pour y avoir part, il faut se mettre à l'œuvre et se placer devant les faits ${ }^{1}$.

3 Si bien que la profusion des traces, la puissance insoupçonnable des machines, les effets des ruptures démographiques sur la transmission du métier de savant dans chaque science, l'irruption enfin de formes nouvelles d'organisation et de financement sur les ruines d'institutions vieilles d'à peine quelques décennies, tout cela a profondément altéré les conditions concrètes - sociales et donc intellectuelles et théoriques entend le sociologue - de l'acquisition des gestes proprement scientifiques.

Chacun le ressent, partagé entre un nécessaire attachement à cette âme que Durkheim reconnaissait dans la conscience des savants et une curiosité, non moins fondée, que toute situation nouvelle ne peut manquer d'éveiller dans la même conscience. Ce moment n'est pas confortable, et tout porte à croire qu'il va durer. Les uns, opportunistes, dansent 
sur l'air de la liquidation en croyant s'emparer des dépouilles. D'autres, craignant de perdre l'essentiel, entretiennent le souvenir ancien d'une science éternelle. À ce point, le sociologue - ou l'historien des sciences attentif aux acquis des sciences sociales et de l'historiographie générale - peut apporter quelques éléments de réflexion.

5 Des conjonctures comparables ont en effet scandé le $\mathrm{xx}^{\mathrm{e}}$ siècle ${ }^{2}$. Ce fut tout d'abord, pendant les années 1908-1910, l'intense débat sur la nouvelle Sorbonne. Les tenants du modèle scientifique dans l'apprentissage des disciplines qui relevaient jusqu'alors de la transmission des humanités affrontaient les déclinologues du moment. Ce furent ensuite les recompositions sur le champ de ruines laissé par la Première Guerre mondiale, à Paris sur la Montagne Sainte-Geneviève, mais aussi sous l'égide de la nouvelle Université de Strasbourg, où une génération de jeunes professeurs parmi lesquels notamment le mathématicien Maurice Fréchet, les historiens Lucien Febvre et Marc Bloch ou le sociologue Maurice Halbwachs ont inventé ce que nous appelons aujourd'hui l'interdisciplinarité ${ }^{3}$. Ce furent enfin pour les sciences sociales et l'histoire, à l'issue du second conflit mondial, la création de la $\mathrm{VI}^{\mathrm{e}}$ section de l'École des Hautes Études qui prit ensuite son autonomie sous la forme de l'École des Hautes Études en Sciences Sociales ${ }^{4}$.

6 À chaque occasion, des générations de savants se sont soudées autour d'identités institutionnelles et intellectuelles collectives; des avant-gardes ont exploré et découvert des voies originales vers de nouvelles confrontations et transactions entre des disciplines établies, mais aussi des arrière-gardes non moins productrices; de profonds remaniements de la mémoire collective ont eu lieu dans chaque discipline, c'est-à-dire au cas par cas de nouvelles manières de penser pertinemment son action savante, de reconsidérer celles de ses prédécesseurs et de concevoir celles de ses successeurs possibles 5 .

7 Si bien qu'aujourd'hui la question de la conduite des sciences dans la décennie à venir n'est pas tant de savoir si elles seront capables de traverser la zone de fortes turbulences dans laquelle elles sont déjà entrées, car elles le feront, dussent-elles y perdre beaucoup. Elle est de savoir comment elles peuvent le faire en demeurant attachées à ce que Bachelard appelait "l'action décisive de la raison", étant entendu que depuis un siècle nous avons pris l'habitude de voir cette question réglée de fait, au rythme des catastrophes planétaires qui ont décimé ou remodelé les cohortes de savants, au détriment ou au bénéfice d'aspects particuliers de l'activité scientifique. En d'autres termes, il est possible d'avancer cette conjecture: du fait qu'au long $d u x^{e}$ siècle les scientifiques de toutes disciplines ont rencontré la question de la pérennisation des sciences sous l'emprise de ruptures démographiques tragiques et répétées, ils sont aujourd'hui passablement démunis face aux bouleversements contemporains, tant ceuxci relèvent sur le plan démographique de renouvellements banals et, parallèlement, sur les plans technologiques ou institutionnels de ruptures à peine concevables.

8 Tout porte ainsi à considérer que, au $\mathrm{xx}^{\mathrm{e}}$ siècle et dans les milieux scientifiques les plus divers - en mathématique, en physique, en biologie comme en sciences sociales, la distinction entre ces domaines n'étant pas la question, même si les modalités diffèrent ici ou là -, l'expérience collective de la science est caractérisée par un affaiblissement de la conscience des formes particulières de la durée des sciences et, par voie de conséquence, aux premières décennies du siècle suivant, par une périlleuse candeur qui laisse les savants désarmés, comme perdus entre liquidation et régression scolastique. Sans doute convient-il alors de réévaluer les manières plus anciennes qu'avaient les savants, au XVIII ${ }^{\mathrm{e}}$ et au $\mathrm{XIX}^{\mathrm{e}}$ siècle, de se prémunir contre ces deux périls et de reconsidérer ce que la 
sociologie qualifie de conditions de l'autonomie relative de leur action spécifique ${ }^{6}$. C'est une première voie.

9 On peut aussi, l'un n'empêchant pas l'autre au demeurant, aller vers deux auteurs déjà cités ici et qui ont abordé par des voies différentes ce genre de questions dans le contrecoup de la Première Guerre mondiale. Le sociologue Maurice Halbwachs, avec sa théorie de la mémoire collective, a entendu explorer une sociologie de la pérennisation des cadres de la pensée. Le philosophe Gaston Bachelard, de son côté, répondait en 1936 aux premières lignes du premier numéro de La Révolution surréaliste au moyen du manifeste « Le Surrationalisme », qui débutait ainsi ${ }^{7}$ :

On confond presque toujours l'action décisive de la raison avec le recours monotone aux certitudes de la mémoire. Ce qu'on sait bien, ce qu'on a expérimenté plusieurs fois, ce qu'on répète fidèlement, aisément, chaleureusement, donne une impression de cohérence objective et rationnelle. Le rationalisme prend alors un petit goût scolaire. Il est élémentaire et pénible, gai comme une porte de prison, accueillant comme une tradition. [...] Bref, il faut rendre à la raison humaine sa fonction de turbulence et d'agressivité. On contribuera ainsi à fonder un surrationalisme qui multipliera les occasions de penser. Quand ce surrationalisme aura trouvé sa doctrine, il pourra être mis en rapport avec le surréalisme, car la sensibilité et la raison seront rendues, l'une et l'autre, ensemble, à leur fluidités.

Halbwachs et Bachelard nous paraissent ainsi procurer des points de départ solides en vue d'une réélaboration scientifique, d'une reconstruction à vrai dire, des critères d'un travail raisonné qui porterait sur la longue durée des sciences et qui serait conduit par les sciences elles-mêmes, et cela non seulement en philosophie et en sciences sociales mais aussi dans les autres disciplines.

11 Ainsi, par exemple, ce sont des renouvellements de la manière de procéder en histoire des mathématiques qui peuvent conduire les mathématiciens eux-mêmes, non seulement à la réévaluation de textes mathématiques anciens, mais encore à un réexamen des formes jusque là admises de la transmission de textes canoniques. L'exemple en est donné par la réception récente au sein de la communauté mathématique des travaux de Caroline Ehrhardt sur Galois et sur sa postérité, recherches conduites au plus près de l'état des savoir-faire en vigueur aujourd'hui dans l'historiographie générale (renouvellements de la micro-histoire et des enquêtes de biographie intellectuelle) et dans la sociologie générale (apports de la sociologie de la mémoire collective).

En suivant une autre ligne, on peut entreprendre des enquêtes d'épistémologie, d'histoire ou de sociologie des constructions savantes anciennes, procédant ainsi à une déconstruction systématique, jusqu'à atteindre les points de résistance des objets anciennement étudiés et, sur ces points, à édifier de nouvelles constructions, certes anachroniques mais dont l'ajustement aux formes de résistance anciennes de l'objet est rationnellement contrôlée ${ }^{10}$.

13 Toutefois plusieurs écrans s'interposent qui entravent l'exploration d'un tel programme réaliste et expérimental d'examen et d'approfondissement de l'expérience collective de la durée des sciences. Ils proviennent précisément de ce que l'on a excessivement radicalisé, dans le contexte du double bouleversement de la physique au début du $\mathrm{xx}^{\mathrm{e}}$ siècle, le constat et le diagnostic des ruptures épistémologiques. De révolution einsteinienne en révolution copernicienne, le geste de Bachelard ${ }^{11}$, ou bien celui de Koyré ${ }^{12}$, amplifié, conduisit chez Kuhn jusqu'aux " paradigmes $»^{13}$ et chez Foucault jusqu'aux «épistémés ${ }^{14}$ ", ces deux auteurs, et ceux qui les ont suivis, ayant eu ensuite à se débattre sans répit avec l'épineuse question de l'incommensurabilité entre deux époques des sciences ${ }^{15}$. 
Plusieurs tentatives de réponses ont été apportées. Outre la thèse de l'incommensurabilité radicale qui court à l'état de vulgate, on a cherché des échappatoires par la logique (logique formelle ou logique des sciences), d'autres par la trame des contextes sociaux et historiques, quitte à importer en épistémologie les fragments les plus commodes que paraissaient procurer la sociologie ou l'ethnologie, mais sans assumer les exigences d'une sociologie générale dont les concepts ne se laissent pas si facilement instrumentaliser ${ }^{16}$. Pire, l'instrumentalisation des sciences sociales fut comme une porte grandement ouverte devant des thèses qui, quant au fond, consistaient à ratiociner les tendances de la politique publique des sciences ${ }^{17}$.

On ne sort de ces débats où des fragments de disciplines sont réquisitionnés pour colmater les brèches des autres qu'en procédant à un examen strict des supports de la continuité des sciences. Or ces supports sont de deux ordres. Les uns sont strictement matérialisés : des traces, des choses, des machines ou encore des dispositifs techniques; ils sont "aisément transmissibles", écrivait Durkheim, extériorisables si ce n'est extériorisés ${ }^{18}$. Les autres sont intérieurs aux savants: alors "tout est personnel», précisait-il. Voilà bien les deux trames sur lesquelles l'histoire et l'action se déroulent pour des sociologues généralistes tels Halbwachs ou Bourdieu, en particulier l'histoire des sciences et l'action de la raison. Ce sont ici les deux prises empiriques sur lesquelles il faut construire.

16 Aussitôt un premier constat s'impose : les durées engagées dans ces deux registres (celui des traces extérieures aux agents et celui des élaborations intérieures) n'ont rien de commun. Elles ne se conjuguent que sous l'emprise de dispositifs matériels savamment construits (des instruments par exemple, ou des formules) ou de dispositifs conceptuels pensés plutôt qu'écrits mais non moins solides en principe. Voici qui caractérise des moments scientifiques particuliers - des instants souvent mais aussi bien des époques - et qui permet de saisir comment ils peuvent être transportés ultérieurement.

17 Par suite la différentiation des sciences et leurs recompositions relèvent à strictement parler de ces remaniements de la mémoire collective spécialisée conçus par Halbwachs. On distingue dans son œuvre d'une part l'analyse des remaniements de traditions qui imposent, selon ce sociologue, un travail d'entretien des doctrines. Il y a d'autre part des remaniements de lieux, c'est-à-dire, pour ce qui nous concerne ici dans le monde scientifique, celui des corpus, des machines, des édifices antérieurs et des objets de la recherche. Enfin Halbwachs constate la nécessité de transactions, tant ces remaniements ne sont pas gratuits et tant ils imposent des échanges entre des détenteurs antérieurement séparés ${ }^{19}$. À l'échelle de siècles, si une mémoire collective perdure, c'est parce qu'elle est nécessairement morcelée, parce qu'elle peut aller et venir d'un lieu à un autre, être portée par des éléments distincts de la tradition, et ces migrations et ces tensions permanentes, un durkheimien les aurait qualifiées de «vie collective» de la mémoire des disciplines concernées.

18 Sans doute la sociologie générale apporte-t-elle à la réflexion que ne peut manquer de susciter l'ébranlement actuel des sciences bien autre chose que ce que l'ignorance fait paresseusement attendre d'elle - des recettes, des bribes de théories, des trucs de communication ou des doctrines de management. En effet, la sociologie contribue ici à faire prendre conscience de la manière dont les scientifiques eux-mêmes, et selon leurs critères propres, peuvent se réapproprier les formes particulières de la durée de leur expérience collective. 


\section{NOTES}

1. Émile Durkheim, De la division du travail social, Paris, [Alcan, 1893] PUF, 1978, p. 354.

2. Suivre plus d'un siècle durant les prises de positions émises dans la Revue de synthèse se révèle ici instructif. Pour un parcours, voir Éric Brian, «Cent dix ans de renouvellements incessants. Note sur l'itinéraire de la Revue de synthèse de 1900 à 2010 ", Revue de synthèse, t. 131, nº 3, 2010, p. 401-438.

3. En témoignent aussi bien des ouvrages issus de collaborations inédites - tels Lucien rebvre et Albert Demangeon, Le Rhin, Strasbourg, Société générale alsacienne de banque, 1931, ou Maurice $\mathrm{F}$ réchet et Maurice Halbwachs, Le Calcul des probabilités à portée de tous, Paris, Dunod, 1924 - que les comptes-rendus des "Réunions du samedi » publiés dans le Bulletin de la Faculté des Lettres de Strasbourg, Librairie Istra, 1922 et années suivantes; ou encore certains des premiers volumes de l'Encyclopédie française (1935-1939) qu'a dirigée Lucien Febvre lui-même.

4. Voir notamment Brigitte Mazon, Aux origines de l'École des hautes études en sciences sociales : le rôle du mécénat américain 1920-1960, Paris, Le Cerf, 1988 et Jacques Revel et Nathan wachtel (dir.), Une École pour les sciences sociales: de la VI ${ }^{e}$ Section à l'École des hautes études en sciences sociales, Paris, Le Cerf, 1996.

5. Le principe de tels remaniements des mémoires collectives spécialisées, et notamment scientifiques, est un pan important de la sociologie de la mémoire inventée par Maurice Halbwachs pendant l'entre-deux-guerres, œuvre qu'on a redécouverte depuis une quinzaine d'années et qui connaît un retentissement mondial considérable tant le $\mathrm{xx}^{\mathrm{e}}$ siècle a laissé partout dans le monde des blessures irréparables, et tant (il faut le redire) les technologies de l'information des dernières décennies ont bouleversé nos capacités à mobiliser la matière du travail collectif de la mémoire : dépôts documentaires, mutation des pratiques et du concept d'archive, omniprésence des images anciennes, animées ou non, colorées ou non. Pour une réévaluation, voir Maurice Halbwachs, La Topographie légendaire des évangiles en terre sainte. Étude de mémoire collective [1 ${ }^{\text {ère }}$ éd. 1941], éd. Marie Jaisson, Paris, PUF, 2008.

6. Sur ce cadre d'analyse, voir tout particulièrement Pierre Bourdieu, Les Règles de l'art: genèse et structure du champ littéraire, Paris, Le Seuil, 1992.

7. Elles étaient parues en décembre 1924, douze ans plus tôt: «Le procès de la connaissance n'étant plus à faire, l'intelligence n'entrant plus en ligne de compte, le rêve seul laisse à l'homme tous ses droits à la liberté. »

8. Gaston Bachelard, «Le Surrationalisme », Inquisitions, 1936 (rééd. Paris, CNRS, 1990). Plusieurs numéros de la Revue de synthèse, issus du Laboratoire disciplinaire de Charles Alunni à l'École normale supérieure (t. $120, \mathrm{n}^{\circ} 1,1999 ;$ t. $122, \mathrm{n}^{\circ} 1,2001 ;$ t. 126, $\left.\mathrm{n}^{\circ} 2,2005\right)$, ont réexaminé et réévalué cet agenda surrationaliste. Il est pertinent de l'articuler avec les propositions de Gilles $c$ hâtelet, Les Enjeux du mobile. Mathématique, physique, philosophie, Paris, Le Seuil, 1993, et id., L'Enchantement du virtuel. Mathématique, physique, philosophie, éd. Charles Alunni et Catherine $\mathrm{P}$ aoletti, Paris, éditions Rue d'Ulm, 2010.

9. Voir Caroline Ehrhardt, Évariste Galois et la théorie des groupes. Fortune et réélaborations (1811-1910), Paris, thèse de l'EHESS, 2007.

10. En voici trois exemples qui plaident chaque fois pour une réévaluation du caractère stochastique des phénomènes économiques et sociaux : en démographie historique, Éric Brian, «Nouvel essai pour connaître la population du royaume. Histoire des sciences, calcul des probabilités et population de la France vers 1780 ", Annales de démographie historique, n²001-2, 
p. 173-222 ; en économie, id., Comment tremble la main invisible. Incertitude et marchés, Paris, Springer, 2009 ; et en sociologie générale, id. et Marie Jaisson, Le Sexisme de la première heure. Hasard et sociologie, Paris, Raisons d'agir, 2007.

11. Gaston Bachelard, La Valeur inductive de la relativité, Paris, Vrin, 1929.

12. Alexandre Koyré, From the Closed World to the Infinite Universe, Baltimore, Johns Hopkins Press, 1957.

13. Thomas S. Kuhn, The Structure of Scientific Revolutions, Chicago, The University of Chicago Press, 1962.

14. Michel foucault, Les Mots et les choses. Une archéologie des sciences humaines, Paris, Gallimard, 1966.

15. La question est posée avec finesse dans le quatrième chapitre de Mario Biagioli, "The Anthropology of Uncommensurability ", in Galileo, Courtier. The Practice of Science in the Culture of Absolutism, Chicago, The University of Chicago Press, 1993.

16. David Bloor, Knowledge and Social Imagery, Londres, Routledge \& Kegan Paul, 1976; les différentes propositions des auteurs de Science as Practice and Culture, ed. Andrew Pickering, Chicago, The University of Chicago Press, 1992 ; Steven shapin and Simon schaffer, Leviathan and the Air-Pump : Hobbes, Boyle, and the Experimental Life, Princeton, Princeton University Press, 1985.

17. Terry shinn, "Change or Mutation? Reflections on the Foundations of Contemporary Science ", Social Science Information, vol. 38, n 1, March 1999, p. 149-176, qui analyse les modèles de la « Nouvelle production du savoir » et de la « Triple hélice».

18. Ce constat rejoint celui de Peter Galison, Image and Logic. A Material Culture of Microphysics, Chicago, The University of Chicago Press, 1997.

19. Ici encore on peut rejoindre Galison qui toutefois ne se situe pas dans ce cadre analytique halbwachsien. Pour aller plus loin dans ce registre conceptuel, voir Éric Brian, « Portée du lexique halbwachsien de la mémoire ", in M. Halbwachs, La Topographie légendaire..., cité plus haut, p. $113 *-146^{*}$.

\section{RÉSUMÉS}

Alors même que les activités scientifiques connaissent aujourd'hui d'importants bouleversements pratiques, comment la sociologie peut-elle contribuer à favoriser à la fois la préservation des acquis scientifiques et le renouvellement des combinatoires disciplinaires? Il importe de réviser les notions de rupture, de continuité et de durée telles qu'on peut les mettre en œuvre à propos des sciences et de les considérer du point de vue de la sociologie générale de la mémoire collective.

Considering that Science is now experiencing major practical upheavals, how can sociology contribute to promoting both the preservation of relevant scientific developments and the renewal of disciplinary combinations? It is time to revise the notions of rupture, continuity and duration such as they are being applied to Science and to consider them from the standpoint of the general Sociology of collective memory. 
INDEX

Mots-clés : rupture, paradigme, mémoire collective, sciences du xxe siècle

Keywords : rupture, paradigm, collective memory, science of the 20th Century

\section{AUTEUR}

\section{ÉRIC BRIAN}

Centre Maurice-Halbwachs (ENS-EHESS-CNRS)

Directeur d'études à l'EHESS, historien des sciences et sociologue.

Parmi les publications :

En collaboration avec M. Jaisson. Le Sexisme de la première heure. Hasard et sociologie, Paris, Raisons d'agir, 2007.

Comment tremble la main invisible. Incertitude et marchés, Paris, Springer, 2009.

Il dirige la Revue de synthèse. 